\title{
NOTES SUR LA DISTRIBUTION \\ CONDITIONNEE DU MONTANT D'UN SINISTRE PAR RAPPORT A L'HYPOTHESE QU'IL Y A EU UN SINISTRE DANS L'ASSURANCE AUTOMOBILE
}

\author{
GunNar Benktander, \\ Solna (Suède)
}

Dans un traité antérieur, l'auteur a examiné, en collaboration avec M. Segerdahl, un certain nombre de fonctions analytiques propres à définir la distribution du montant d'un sinistre en s'arrêtant tout spécialement aux aspects présentant de l'intérêt du point de vue de l'assurance en excédent de sinistres. L'examen a révélé que les répartitions du type Pareto étaient intéressantes aussi bien du point de vue théorique - en tant que représentant un type de distribution particulièrement ,dangereux" - que du point de vue pratique, le matériel statistique suédois et norvégien obtenu lors de l'analyse de différentes branches d'assurances - telles que Vie, Incendie et Automobile - semblant bien indiquer que pour de grands intervalles la distribution du type Pareto représenterait l'authentique matériel des dommages.

Nous nous permettrons par conséquent de nous arrêter tant soit peu aux caractéristiques de la distribution du type Pareto ainsi qu'aux formules découlant de la dite distribution pour la prime de risque en excédent de sinistres et ses variances.

Si $P(x)$ exprime la distribution, I $-P(x)=H(x)=C \cdot x^{-\alpha}$ pour $a<x \leq M, M$ étant la valeur la plus grande pour $x$, en supposant que la probabilité d'atteindre la valeur $M$ est $H(M)=C \cdot M^{-\alpha}$.

Si $m(x)$ est la moyenne des portions de sinistres supérieures à $x$

$$
m(x)=\frac{x}{\alpha-\mathrm{I}}\left\{\mathrm{I}-\left(\frac{x}{M}\right)^{\alpha-1}\right\}
$$

Spécialement si $M=\infty$ on obtient $m(x)=\frac{x}{\alpha-\mathrm{I}}$.

Si nous appelons $n(x)$ le nombre prévu de sinistres supérieurs à $x$ 
pendant une période donnée, et $\Pi(x, M)$ la prime de risque pour un traîté d'excédent de sinistre correspondant à la dite période avec une priorité $x$,

$$
\Pi(x, M)=n(x) \cdot m(x)=n . H(x) \cdot m(x),
$$

$n$ étant mis pour $n(O)$.

On obtient donc dans le cas Pareto

$$
\Pi(x, M)=\Pi(x)-\Pi(M)=n \cdot C \cdot x^{-\alpha} \cdot \frac{x}{\alpha-\mathrm{I}}\left\{\mathrm{I}-\left(\frac{x}{M}\right)^{\alpha-1}\right\},
$$

où $\Pi(x)$ est mis pour $\Pi(x, \infty)$, soit la prime de risque pour la tranche illimitée.

Si $M=k \cdot x$, on obtient $\Pi(x, k x)=\Pi(x) \cdot\left(I-k^{-\alpha+1}\right)$

Spéc. I $\quad \alpha=2 \quad \Pi(x, k x)=\Pi(x) \cdot\left(\mathrm{I}-\frac{\mathrm{I}}{k}\right)$

Spéc. $2 \quad \alpha=3 \quad \Pi(x, k x)=\Pi(x) \cdot\left(\mathrm{I}-\frac{\mathrm{I}}{k^{2}}\right)$

En supposant des facteurs de risque constants et des sinistres indépendants l'un de l'autre, la variance de la somme du montant des sinistres venant à frapper la couverture en excédent de sinistres $(x, M)$ sera

$$
V(x, M)=2 \int_{x}^{M} \Pi(y) d y-2(M-x) . \Pi(M)
$$

En supposant que $V(x)=V(x, \infty)$ existe, c.à.d., dans le cas Pareto, que $\alpha>2$, on peut écrire

$$
V(x, M)=V(x)-V(M)-2(M-x) \Pi(M)
$$

Lorsque $\alpha=2$ et $\Pi(x)=n . C \cdot x^{-1}=A_{2} \cdot x$, on obtient

$$
V(x, M)=2 A_{2}\left\{\log \frac{M}{x}-(M-x) \cdot \frac{\mathrm{I}}{M}\right\} \quad \text { et }
$$

$V(x, k x)=2 A_{2}\left\{\log k-\left(\mathrm{I}-\frac{\mathrm{I}}{k}\right)\right\}=2 x . \Pi(x) \cdot\left\{e \log k-\left(\mathrm{I}-\frac{\mathrm{I}}{k}\right)\right\}$.

Par conséquent, les variances sont ici égales pour toutes les tranches ayant la même longueur relative $k$.

Lorsque $\alpha=3$ et $\Pi(x)=A_{3} \cdot x^{-2}$, on obtient 


$$
\begin{aligned}
& V(x, M)=2 A_{3}\left\{\frac{\mathrm{I}}{x}-\frac{\mathrm{I}}{M}-(M-x) \cdot \frac{\mathrm{I}}{M^{2}}\right\}=2 A_{3} \cdot \frac{\mathrm{I}}{x}\left(\mathrm{I}-\frac{x}{\bar{M}}\right)^{2} \text { et } \\
& V(x, k x)=2 A_{3} \cdot \frac{\mathrm{I}}{x}\left(\mathrm{I}-\frac{\mathrm{I}}{\bar{k}}\right)^{2}=2 x \cdot \Pi(x)\left(\mathrm{I}-\frac{\mathrm{I}}{\bar{k}}\right)^{2}
\end{aligned}
$$

Dans l'application pratique, les cas présentant un intérêt plus particulier sont ceux où $\alpha>2$, c.à.d. où le résultat de la tranche illimitée a une variance limitée.

Pour illustrer l'utilité des distributions du type Pareto dans la branche Accidents nous avons choisi et remanié un certain matériel provenant d'un pays européen non scandinave et relatif à l'assurance $\mathrm{RC}$ Automobiles couvrant la période r955-58. Les sinistres particuliers ont été recalculés en tenant compte de l'inflation constatée durant la dite période ainsi que d'une certaine ,,inflation dans la jurisprudence" s'ajoutant à la précédente. Par contre, le remaniement n'a pas tenu compte des différences survenues dans le tarif, de la composition du portefeuille, ainsi que des mesures complexes prises en vue de réduire le nombre des sinistres dans leur ensemble. Ces mesures représentent tous les facteurs sur les lesquels l'on s'efforce d'agir au moyen de recherches sur la circulation, de construction de routes, de dispositifs de sécurité à l'intérieur des voitures, de propagande pour la sûreté du trafic, des limites de vitesse, etc.

Le matériel comprend aussi bien les sinistres réglés que les sinistres en suspens. Les compagnies qui semblent avoir systématiquement sousestimé leurs réserves pour sinistres en suspens ont été éliminées de l'étude en question.

Dans la table ci-dessous, une comparaison a été établie entre le nombre de sinistres survenus dans les différentes classes de grandeur et le nombre de sinistres prévus selon la distribution du type Pareto lorsque $\alpha=2.7$.

Intervalle de sinistres en milliers d'unités monétaires

\begin{tabular}{lcc} 
sinistres & \multicolumn{2}{c}{ nombre de sinistres } \\
survenus & théoriques \\
I75-200 & IO6 & 93 \\
$200-25^{\circ}$ & IOI & 97 \\
$250-300$ & 3 I & 46 \\
$300-350$ & 24 & 25
\end{tabular}


Intervalle de sinistres

en milliers d'unités monétaires

$$
\begin{aligned}
& 35^{0}-400 \\
& 400-45^{\circ} \\
& 45^{0}-500 \\
& 500-55^{\circ} \\
& 550-700 \\
& 700-
\end{aligned}
$$

nombre de sinistres survenus théoriques

\begin{tabular}{rr} 
I0 & I4 \\
I2 & 9 \\
8 & 6 \\
4 & 4 \\
5 & 7 \\
7 & 7 \\
\hline 308 & 308
\end{tabular}

A part le troisième intervalle de sinistres, la correspondance est excellente. $\chi^{2}=$ ro.3 et le nombre de degrés de liberté est 8 .

La bonne correspondance est également illustrée par le diagramme ci-dessous, dans lequel on a comparé les valeurs observées de la fonction avec les valeurs théoriques de la même fonction.

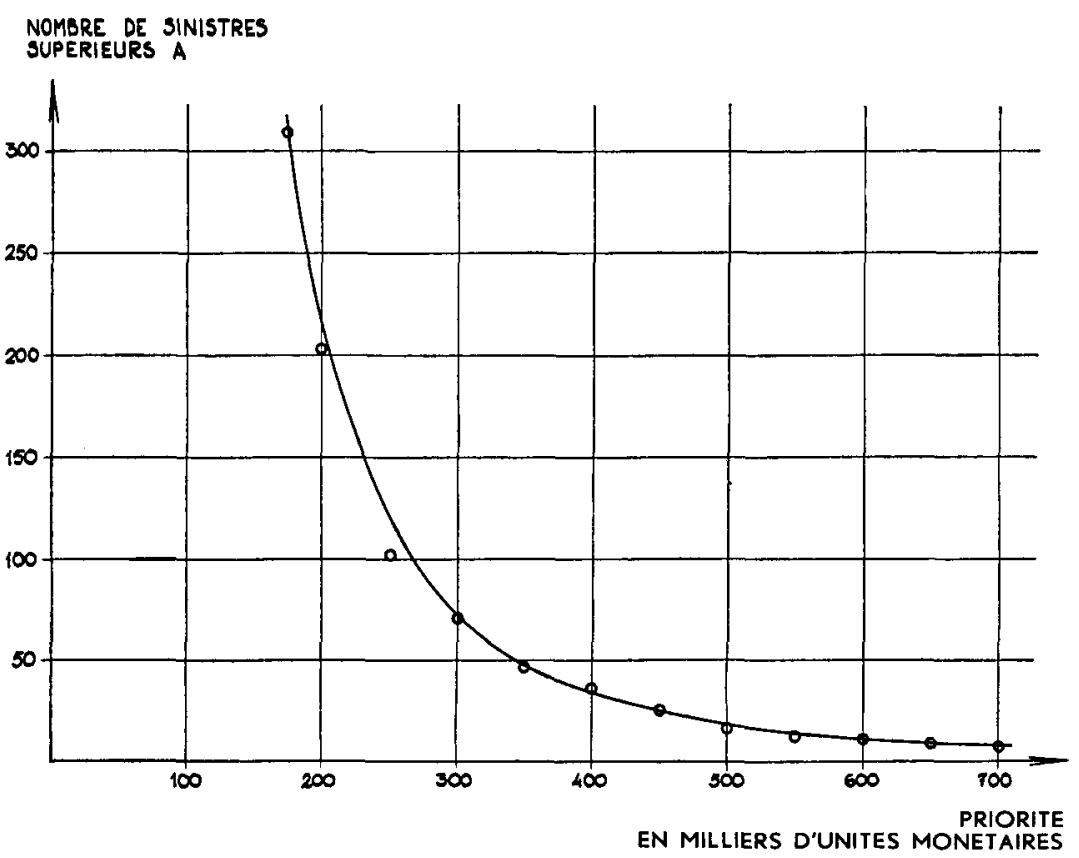

Mais à la priorité $x$ la prime en excédent est égale au produit du nombre prévu de sinistres $n(x)$ et du chiffre moyen prévu des sinistres en excédent $m(x)$. Dans la table ci-après, on a donc comparé 
le chiffre moyen des sinistres en excédent survenus avec les valeurs théoriques basées sur la distribution du type Pareto lorsque $\alpha=2.7$.

Intervalle de sinistres moyenne de sinistres en excédent en milliers d'unités monétaires

$\begin{array}{rr}\text { valeurs } & \text { valeurs } \\ \text { observées } & \text { théoriques }\end{array}$

I $75-700$
$200-700$
$250-700$
$300-700$
$350-700$
$400-700$
$450-700$
$500-700$
$550-700$

$\begin{array}{rr}89 & 93 \\ \text { I05 } & \text { I04 } \\ \text { I37 } & \text { I22 } \\ \text { I38 } & \text { I35 } \\ \text { I47 } & \text { I43 } \\ \text { I33 } & \text { I45 } \\ \text { I33 } & \text { I40 } \\ \text { I38 } & \text { I28 } \\ \text { I25 } & \text { I09 }\end{array}$

Pour les intervalles supérieurs à 700.000 unités monétaires où les sinistres sont rares, la correspondance est moins bonne, ce qui peut toutefois être attribué au hazard.

Pour la prime de risque en excédent de sinistres, finalement, on obtient les résultats-normes suivants:

Tranches en milliers

d'unités monétaires

I 75-700

200-700

250-700

$300-700$

$350-700$

400-700

$450-700$

$500-700$

$550-700$
Primes en excédent de risque valeur observée valeur théorique

$\begin{array}{rr}\text { I00 } & \text { I00 } \\ 77 & 78 \\ 50 & 50 \\ 35 & 34 \\ 25 & 23 \\ \mathrm{I} 7 & 16 \\ \mathrm{I} 2 & 12 \\ 8 & 8 \\ 5 & 5\end{array}$

Les résultats obtenus sont avant tout valables pour l'intervalle qu'ils représentent, soit 175.000 à 700.000. Un extension de la validité de la fonction hors de cet intervalle doit être envisagée avec la plus grande prudence. Toutefois, une extension vers le haut, soit vers les tranches supérieures, particulièrement faibles en sinistres, 
peut être considérée comme permise et représenterait même la meilleure évaluation possible du risque. Une extension vers le bas, c'est-à dire vers les tranches inférieures à 175.000 , riches en sinistres, ne peut être effectuée sans plus. En lieu et place, il convient d'étudier les tranches en question en fonction des statistiques y relatives, mais celles-ci n'ont pas été à la disposition de l'auteur.

\section{RÉFERENCE:}

"On the Analytical Representation of Claim Distributions with Special References to Excess of Loss Reinsurance (by Gunnar Benktander and CarlOtto Segerdahl, Sweden). - The XVIth International Congress of Actuaries, Brussels, I960." 